\title{
Optimally segmented permanent magnet structures
}

Insinga, Andrea Roberto; Bjørk, Rasmus; Smith, Anders

Published in:

I E E E Transactions on Magnetics

Link to article, DOI:

10.1109/TMAG.2016.2593685

Publication date:

2016

Document Version

Peer reviewed version

Link back to DTU Orbit

Citation (APA):

Insinga, A. R., Bjørk, R., \& Smith, A. (2016). Optimally segmented permanent magnet structures. I E E E Transactions on Magnetics, 52(12), [7210306]. https://doi.org/10.1109/TMAG.2016.2593685

\section{General rights}

Copyright and moral rights for the publications made accessible in the public portal are retained by the authors and/or other copyright owners and it is a condition of accessing publications that users recognise and abide by the legal requirements associated with these rights.

- Users may download and print one copy of any publication from the public portal for the purpose of private study or research.

- You may not further distribute the material or use it for any profit-making activity or commercial gain

- You may freely distribute the URL identifying the publication in the public portal

If you believe that this document breaches copyright please contact us providing details, and we will remove access to the work immediately and investigate your claim 


\title{
Optimally segmented permanent magnet structures
}

\author{
A. R. Insinga*, R. Bjørk, A. Smith and C. R. H. Bahl \\ Department of Energy Conversion and Storage \\ Technical University of Denmark \\ Frederiksborgvej 399, 4000 Roskilde, Denmark. \\ *Email: aroin@dtu.dk
}

\begin{abstract}
We present an optimization approach which can be employed to calculate the globally optimal segmentation of a two-dimensional magnetic system into uniformly magnetized pieces. For each segment the algorithm calculates the optimal shape and the optimal direction of the remanent flux density vector, with respect to a linear objective functional. We illustrate the approach with results for magnet design problems from different areas, such as a permanent magnet electric motor, a beam focusing quadrupole magnet for particle accelerators and a rotary device for magnetic refrigeration.
\end{abstract}

\section{Introduction}

With the development of new and powerful magnetic materials [1], the range of application of permanent magnets is expanding to many scientific and technological areas [2]. The geometry of the magnetic system must be optimized to achieve high efficiency and minimize the amount of permanent magnetic material. Among the wide range of geometry optimization techniques we can identify two important categories of algorithms: topology optimization methods and parametric shape optimization methods.

The purpose of topology optimization algorithms is to determine how to subdivide a given design area into regions occupied by materials with different magnetic properties, e.g., magnetic permeability or remanent flux density. Since the topological features of the allowed solution are not restricted by an initial design concept, these algorithms potentially lead to novel geometrical configurations. Typically, the implementation of this class of algorithms employs finite element analysis for the calculation of the magnetic field. The properties of the materials are linked to control fields which are optimized by numerically evaluating the sensitivity of the objective with respect to the control fields $[3,4,5]$ and applying iterative techniques such as sequential linear programming or gradient descent. Alternatively, the control fields can be optimized with gradient-free heuristic approaches, such as genetic algorithms $[6,7,8]$. While being very versatile, topology optimization may produce solutions characterized by finely subdivided or jagged shapes which are not suitable for manufacturing. Avoiding this problem often requires fine tuning of some regularization parameter. Generally, topology optimization techniques present a trade-off between computational time and resolution of the mesh underlying the simulation.

In parametric geometry optimization the shapes of the boundaries between materials with different properties are linked to a finite number of control parameters. The magnetic field for a given configuration may be determined with numerical techniques $[9,10]$, by analytically solving the partial differential equations $[11,12,13]$, or by approximating the magnetic system with a 
simplified model [14]. When numerical methods are employed it is possible to consider arbitrary shapes and to take into account non-linear magnetic effects. With analytical approaches the computational time is very short, but they can only be applied to the geometries for which the solution is available. Moreover, analytical solutions generally assume linear magnetic behavior or even more ideal approximations, such as permanent magnets with permeability equal to one and soft magnetic material with infinite permeability. Parametric optimization techniques are intrinsically limited by the fact that the search space is determined beforehand: the diversity of the allowed configurations is restricted to variations around the same design concept.

The optimization approach discussed in this paper presents some of the advantages of both categories. Since it relies on an analytical result to determine the optimal remanent flux density at any point with a single finite element simulation, it can be implemented in a computationally efficient algorithm. Moreover, it does not share the limitations that are typical for parametric optimization. The method provides the globally optimal subdivision of a given design area into uniformly magnetized segments with respect to a linear objective functional. The optimality is guaranteed as long as each material in the system exhibits a linear magnetic behavior. Under the more restrictive assumption that the permeability of the permanent magnet material is close to 1 we are also able to determine the border between permanent magnet and air which maximizes the linear objective. Another possibility is to optimize the border between permanent magnet and soft magnetic material by considering the limit of infinite permeability.

Since the optimality results are derived under the specific assumptions about the magnetic behavior of the materials in the system, the optimization of magnetic systems which are expected to exhibit a highly non-linear behavior must be performed using other techniques. However, nonlinear magnetic phenomena - such as demagnetization of the permanent magnets due to the finite coercivity and anisotropy field, or magnetic saturation of any soft magnetic materials often cause detrimental effects which it is desirable to minimize. When these effects are expected to be small, it is possible to perform the optimization by assuming linear behavior, and check that the optimized configurations obtained in this way are within the ranges for which the linear approximation is justified. It will be possible to apply small corrections to the geometry with a final optimization step which takes into account the non-linear behavior.

We illustrate the result of our optimization approach with three examples from different fields of application: a permanent magnet electric motor, a beam-focusing quadrupole magnet, and a rotary device for magnetic refrigeration at room temperature. Since the method always assumes a linear objective functional, we will also evaluate the goodness of each optimized configuration in terms of those features of the produced field which are most relevant to the corresponding application. We will also discuss quantitatively the variation of the field when it is computed under more realistic assumptions about the magnetic response of the materials in the system.

\section{Framework of the optimization method}

\subsection{The reciprocity theorem and the Virtual Magnet method}

Our optimization method is based on the reciprocity theorem, an energy equivalence principle of magnetostatics, which can be expressed by the following equation [15]:

$$
\int d V \boldsymbol{B}_{r 1}(\boldsymbol{x}) \cdot \boldsymbol{H}_{2}(\boldsymbol{x})=\int d V \boldsymbol{B}_{r 2}(\boldsymbol{x}) \cdot \boldsymbol{H}_{1}(\boldsymbol{x})
$$

where $\boldsymbol{B}_{r 1}(\boldsymbol{x})$ is the remanence of the system 1 at the point $\boldsymbol{x}, \boldsymbol{H}_{1}$ is the magnetic field generated by $\boldsymbol{B}_{r 1}$, and similarly for system 2 . The integration domain of both the volume integrals extends over the whole space. However, if the integration domain of the left-hand side of (1) was replaced 
with the region where $\left\|\boldsymbol{B}_{r 1}\right\|>0$ the result would be the same, and similarly for the right-hand side. The theorem in this form holds as long as there are no free currents, and all materials in the system obeys a linear $\boldsymbol{B}-\boldsymbol{H}$ relation, i.e.: $\boldsymbol{B}=\underline{\underline{\mu}} \boldsymbol{H}+\boldsymbol{B}_{r}$. The permeability tensor field $\underline{\underline{\mu}}(\boldsymbol{x})$ must be the same for both the systems.

The theorem equates the energy possessed by the magnetic flux sources of system 1 when placed in the field generated by the flux sources of system 2 with the energy possessed by the flux sources of system 2 when placed in the field generated by the flux sources of system 1 . We use this theorem in order to calculate the remanence field $\boldsymbol{B}_{r 1}$ which produces a magnetic field $\boldsymbol{H}_{1}$ which maximizes a given objective. The magnetic system 2 is a mathematical construction that is used to solve this optimization problem. For this reason, the system 1 will also be referred to as the real system and system 2 as the virtual system.

If the virtual remanence $\boldsymbol{B}_{r 2}$ is interpreted as an objective vector field [16, 17], the right-hand side of (1) can be seen as the definition of an objective functional $\mathcal{S}$. Any objective functional which is linear with respect to the field $\boldsymbol{H}_{1}$ can be expressed in this form. Equation (1) implies that the optimal direction of the real remanence $\boldsymbol{B}_{r 1}$, with respect to the objective functional $\mathcal{S}$, is aligned at any point with the virtual field $\boldsymbol{H}_{2}$.

This approach also provides a way to quantify how much a specific point $\boldsymbol{x}$ of the real magnet is contributing to the value of the objective functional $\mathcal{S}$. Once the real remanence has been aligned at the point $\boldsymbol{x}$ with the virtual field $\boldsymbol{H}_{2}(\boldsymbol{x})$, the contribution to the value of $\mathcal{S}$ from the point $\boldsymbol{x}$ is proportional to the norm $H_{2}(\boldsymbol{x})=\left\|\boldsymbol{H}_{2}(\boldsymbol{x})\right\|$. If the relative permeability of the magnet is equal to 1 , the border between magnet and air does not need to be determined in advance: the optimal border lies on a contour level of $\mathrm{H}_{2}$. Since the contribution of any given point to the value of $\mathcal{S}$ is always positive, extending the magnet area can only increase the value of $\mathcal{S}$. This means that being able to determine the magnet border that maximizes $\mathcal{S}$ in presence of a specific constraint on the total magnet volume is equivalent to minimizing the magnet volume that is necessary to obtain a given value of $\mathcal{S}$.

In some cases it is necessary to determine the border separating permanent magnet material from soft magnetic material, such as iron, characterized by such a high permeability that it can be approximated as infinite. In the regions where the permeability is finite, the virtual field $\boldsymbol{H}_{2}$ is normal to the borders with infinite-permeability regions. Being defined as $\boldsymbol{H}_{2}=-\boldsymbol{\nabla} \Phi_{2}$, the virtual field is also normal to the equipotential surfaces of its magnetic scalar potential $\Phi_{2}$. As pointed out in [18], to achieve maximal energy efficiency an equipotential surface of $\Phi_{2}$ can be converted into the boundary between magnet region and highly permeable material so that the magnet is completely shielded from the outside.

\subsection{Optimal Segmentation of $2 \mathrm{D}$ system}

The determination of the virtual field $\boldsymbol{H}_{2}$ provides a continuously varying vector field which at any point gives the optimal direction of the real remanence $\boldsymbol{B}_{r 1}$. In practice, however, magnetic assemblies are realized by splitting the magnet into several uniformly magnetized segments. Because of the linearity, the different points of the magnet are independent, which implies that it is never optimal to split a region over which the direction of the virtual field $\boldsymbol{H}_{2}$ is uniform [19].

For two dimensional systems, the optimal border between two adjacent segments always lies on a contour level of the angle $\psi=\arctan \left(H_{2 y} / H_{2 x}\right)$, reducing the optimal segmentation problem to the simpler one of selecting the optimal contours with a given total number of segments $N_{\text {Segments. }}$.

It can be shown [19] that this problem is equivalent to the problem of approximating a continuous curve $\mathcal{H}(\psi)$ with an inscribed piecewise linear curve, in such a way that the perimeter of the piecewise curve is maximized. The continuous curve to be approximated is parametrized 
by the orientation $\psi$ of the virtual field $\boldsymbol{H}_{2}$. The curve has the dimension of magnetic field integrated over an area and is expressed by the following equation:

$$
\mathcal{H}(\psi)=\int_{R_{\left[\psi_{0}, \psi_{1}\right]}} d S \boldsymbol{H}_{2}(\boldsymbol{x})
$$

The area of the surface element is denoted by $d S$, and the integration domain $R_{\left[\psi_{0}, \psi_{1}\right]}$ is defined as:

$$
R_{\left[\psi_{0}, \psi_{1}\right]}=\left\{\boldsymbol{x}: \psi(\boldsymbol{x}) \in\left[\psi_{0}, \psi_{1}\right]\right\} \quad \cap \quad\{\text { Design Area }\}
$$

The total extension of the design area can be decided arbitrarily depending on the geometrical constraints of each system. The problem of maximizing the perimeter of the piecewise linear approximation of $\mathcal{H}$ can be solved with the desired degree of accuracy by employing dynamic programming [20].

\section{Optimized magnetic systems}

We present magnet design problems from different areas of application and show how our approach can be used to determine the optimally segmented solution. We will express the objective functional for each optimization problem by defining the objective vector field, which corresponds to the remanence $\boldsymbol{B}_{r 2}$ of the virtual system. For each example we will show two figures. The

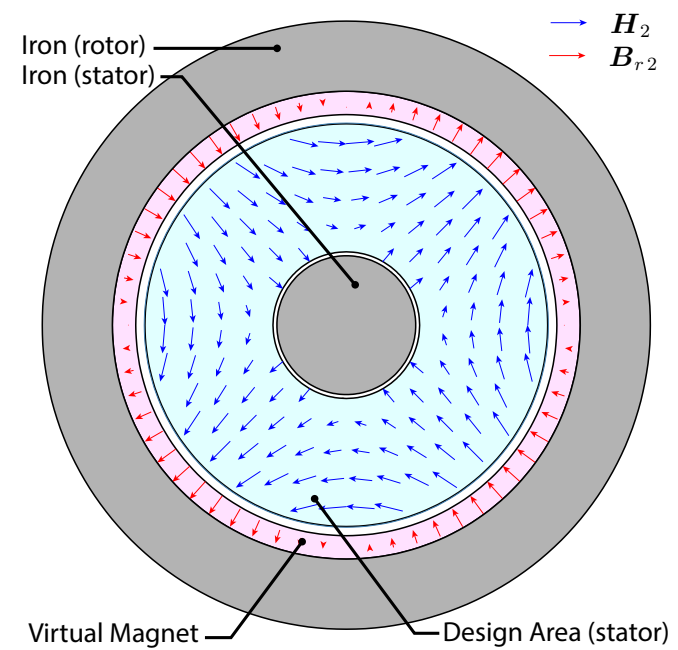

(a)

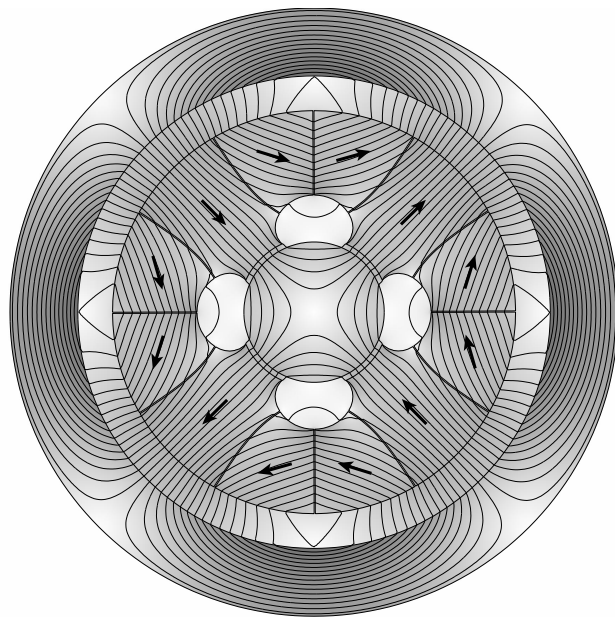

(b)

Figure 1: Designing a magnet for an electric motor with the purpose of creating a sinusoidal radial field in the air gap between the stator and the rotor. Fig. 1(a): geometry of the virtual system. Fig. 1(b): FEM simulation of the optimal segmentation.

first figure (as, e.g., Fig 1(a)) is an illustration of the geometry of the virtual system, including the virtual magnet area, shaded in pink, the design area, shaded in light blue, and the regions originally filled with soft magnetic material, shaded in gray. The virtual remanence vector field is represented by the red arrows, and the virtual field $\boldsymbol{H}_{2}$, which will be shown only inside the design area, is represented by blue arrows.

The second figure (as, e.g., Fig 1(b)) is the result of the Finite Element Method (FEM) simulation for the optimally segmented system performed using the commercial software COMSOL 
Multiphysics. The magnetic field is obtained by projecting on a triangular mesh the partial differential equation governing the magnetic vector potential and solving the resulting system of equations using the direct solver PARDISO, available with the software. The surface area of the mesh elements averaged over the design region is for all the examples $\approx 10^{-5}$ of the surface area of the design region. The real flux density $\boldsymbol{B}_{1}$ is indicated in the figures by the black flux lines, and its norm is indicated by the color, darker shades corresponding to a higher norm. The direction of the optimal remanence for each segment is indicated by a black arrow.

Our approach allows us to choose freely the number of segments $N_{\text {Segments }}$ to be used as a constraint in the optimization. The value of the objective functional will increase monotonically with the number of segments, converging asymptotically to the limit given by the case of a continuously varying remanence field. However, because of the symmetry exhibited by the geometry of each of the example optimization problems, if $N_{\text {Segments }}$ is a multiple of 4 the optimally segmented magnetic system preserves the symmetry, and this results in the additional advantage of many segments having the same shape. We decided to use the value $N_{\text {Segments }}=12$ for all the examples because 3 segments for each symmetric quadrant is a good trade-off between the goodness of the final result and the intent of minimizing the total number of segments which decreases the manufacturing cost of the magnetic assembly.

In all of the examples, the permanent magnetic material has a relative permeability $\mu=1$, and a remanence of $1.4 \mathrm{~T}$. The relative permeability of the soft magnetic material, such as iron, is set equal to $\mu=1000$. The spatial dimensions of the examples will not be reported since the solution is invariant with respect to an isotropic rescaling.

As a final verification step, the field generated by the optimized configuration of each example have been calculated using more realistic models for the magnetic behavior of the materials. In particular, the relative permeability of permanent magnets is set to $\mu=1.05$, and the iron is modeled with the non-linear $B-H$ curve included in the material library of COMSOL, which has a magnetic saturation of $2 \mathrm{~T}$. Moreover, the magnetic field inside the permanent magnet material has been decomposed into the component that is parallel to the remanence and the component that is normal. When the demagnetizing fields or transversal fields are too intense the linear approximation of the $B-H$ relation may not be an accurate description of the magnetic behavior. The parallel and normal components of the field inside the permanent magnet material have been compared with typical values of, respectively, the coercive force and the anisotropy field of typical present-day rare-earth magnets. In all the examples these final validation tests confirmed that the underlying approximations were justified. In cases where this is not true, it will be possible to predict the effect of the non-linear demagnetization of permanent magnets by using numerical approaches such as the one presented in [21].

\subsection{Electric Motor}

The geometry of the virtual system for a four-poles surface-mounted permanent magnet electric motor is shown in Fig. 1(a). The iron core of the stator is surrounded by permanent magnet material. A small air gap is present between the stator and the external iron ring of the rotor (the rotor's slots are not represented). The virtual magnet area is located in the air gap, where the following virtual remanence is defined, with the purpose of minimizing the detrimental higher harmonics [13]:

$$
\boldsymbol{B}_{r 2}=\sin (2 \phi) \hat{\boldsymbol{e}}_{\rho} .
$$

It is possible to apply a constraint on the total volume of permanent magnetic material. We arbitrarily set the volume of magnetic material to $90 \%$ of the total volume of the design region. We use this volume constraint to determine the optimal border between magnet and air by considering the norm of the virtual field $\boldsymbol{H}_{2}$, as explained in section 2.1. This results in the 


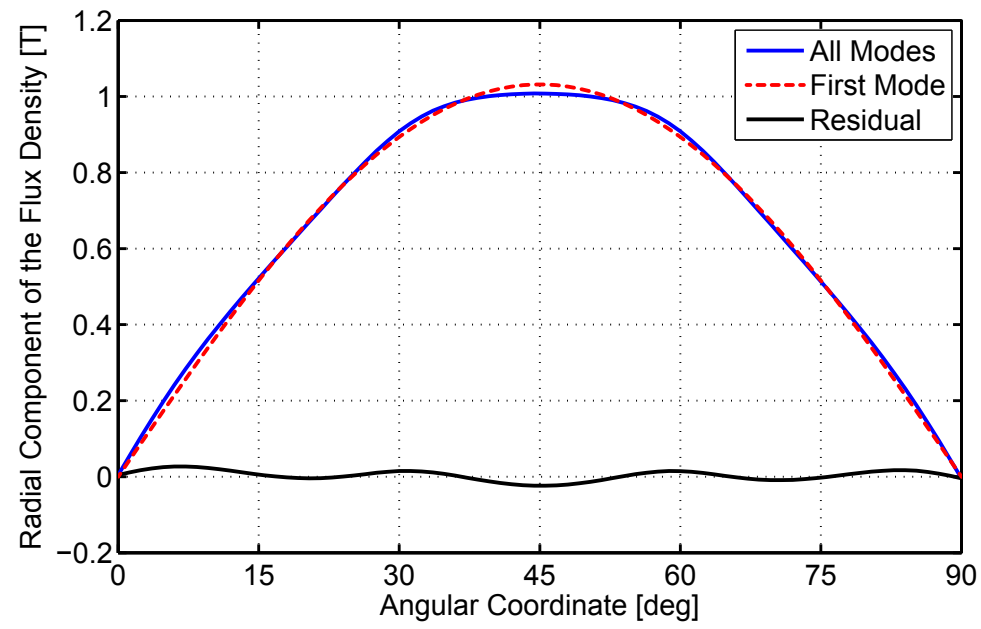

Figure 2: The radial component of the air gap flux density for the electric motor example is plotted as a solid blue line. This curve has been decomposed into the first mode, plotted as a red dashed line, and the sum of all the other undesired harmonics, plotted as a solid black line.

four holes adjacent to the iron core in the center, which are visible in Fig. 1(b). The desired properties of the solution can be checked by expanding in Fourier series the radial component $B_{\rho}$ of the flux density $\boldsymbol{B}_{1}=\mu_{0} \boldsymbol{H}_{1}$ in the air gap between the stator and the rotor, close to external yoke. This is plotted in Fig. 2 for one quadrant of the geometry, given by the following interval of the angular coordinate: $\phi \in\left[0^{\circ}, 90^{\circ}\right]$.

The amplitude of the first harmonic is 0.9998 of the amplitude of the total signal, corresponding to $1.032 \mathrm{~T}$. This implies that the total harmonic distortion, THD, is equal to 0.02. When the field is calculated taking into account the non-linear behavior of iron, and by setting the permeability of the magnets to $\mu=1.05$, the amplitude of the first harmonic decreases to 1.020 $\mathrm{T}$, but remains 0.9998 of the amplitude of the total signal, and thus the THD does not change.

\subsection{Quadrupole magnet for Beam Focusing}

Quadrupole magnets are used in the field of particle acceleration for the purpose of focusing beams of charged particles [22]. The following virtual remanence, corresponding to a quadrupole field, is defined over the square cavity shown in Fig. 3(a):

$$
\boldsymbol{B}_{r 2}=y \hat{\boldsymbol{e}}_{x}+x \hat{\boldsymbol{e}}_{y} .
$$

The magnet area is limited by the external circle visible in the figure. The radius of the circle is determined by the desired field intensity. The results of the FEM simulation for the optimally segmented system is shown in Fig. 3(b). In order to evaluate the optimized magnetic system, we expand the field $\boldsymbol{H}_{1}$ in two components: a perfectly quadrupolar field $\boldsymbol{H}_{Q}(\boldsymbol{x})$, proportional to the virtual remanence $\boldsymbol{B}_{r 2}$ defined in (5), and the residual undesired component of the field, which we denote by $\boldsymbol{\Delta}(\boldsymbol{x})$ :

$$
\boldsymbol{H}_{1}(\boldsymbol{x})=\boldsymbol{H}_{Q}(\boldsymbol{x})+\boldsymbol{\Delta}(\boldsymbol{x})
$$

The field $\boldsymbol{H}_{Q}$ is the second-order term of the interior cylindrical multipole expansion, and $\boldsymbol{\Delta}$ is the sum of all the remaining terms. The calculation of the normalized amplitudes of the two 


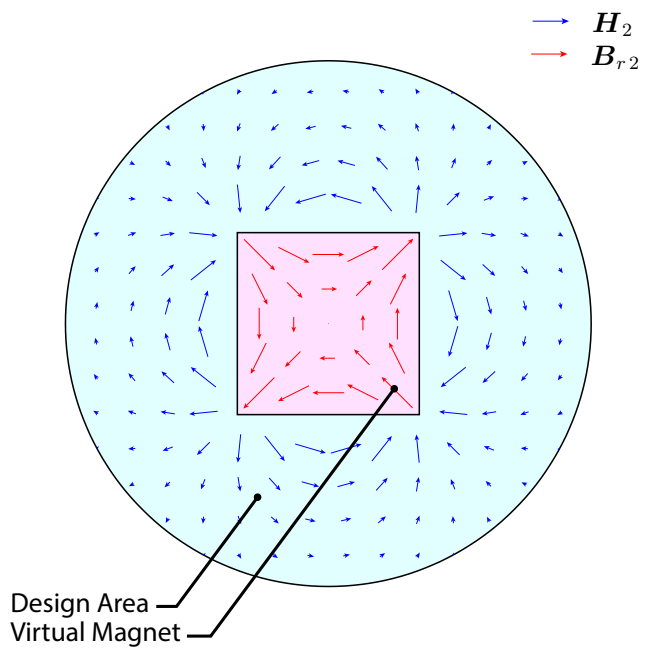

(a)

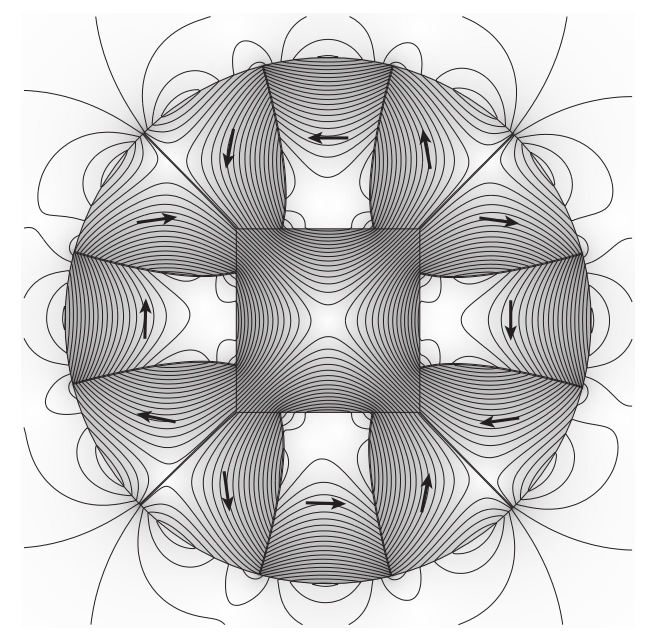

(b)

Figure 3: Design of a quadrupole magnet with a square air gap and circular external border. The design is relevant for beam focusing applications in particle accelerator devices. Fig. 3(a): geometry of the virtual system. Fig. 3(b): FEM simulation of the optimal segmentation.

components shows that the field $\boldsymbol{H}_{1}$ is quadrupolar within a very good approximation:

$$
\begin{aligned}
& c_{Q}=\left(\frac{\int_{\Omega} d S\left\|\boldsymbol{H}_{Q}(\boldsymbol{x})\right\|^{2}}{\int_{\Omega} d S\left\|\boldsymbol{H}_{1}(\boldsymbol{x})\right\|^{2}}\right)^{1 / 2}=0.993 \\
& c_{\Delta}=\left(\frac{\int_{\Omega} d S\|\boldsymbol{\Delta}(\boldsymbol{x})\|^{2}}{\int_{\Omega} d S\left\|\boldsymbol{H}_{1}(\boldsymbol{x})\right\|^{2}}\right)^{1 / 2}=0.120
\end{aligned}
$$

The integration domain $\Omega$ is the whole square cavity. Because of the normalization we have: $c_{Q}^{2}+c_{\Delta}^{2}=0.986+0.014=1$. When the magnetic behavior of permanent magnets and iron is calculated using the more realistic models described at the beginning of section 3 , the values of the coefficients change only slightly, thus giving: $c_{Q}=0.992$ and $c_{\Delta}=0.122$.

\subsection{Magnetic Refrigeration}

Fig. 4(a) shows the geometry of the virtual system for a rotary device for active magnetic refrigeration at room temperature. The following virtual remanence is defined, with the purpose of creating a high field in the pink shaded regions of the air gap and a low field in the angular sectors between them, as desirable for this kind of devices [23]:

$$
\boldsymbol{B}_{r 2}=\operatorname{sign}(x) \hat{\boldsymbol{e}}_{\rho}
$$

where $x$ denotes the coordinate parameterizing the horizontal direction. As shown in Fig. 4(a), the virtual remanence is only defined in the high field regions of the air gap, which are situated on the two sides of the iron core.

We apply a constraint on the total volume of magnet material, which is equal to 5 times the volume of the high field region. This ratio is comparable to other published designs of magnetic refrigeration devices [24]. 
As explained in section 2.1, we can convert an equipotential line of the virtual scalar potential $\Phi_{2}$ into the external border between permanent magnet and soft magnetic material. The level curves corresponding to the volume constraint are the kidney shaped lines shown on both the sides of the air gap in Fig. 4(b). After determining the boundary between magnet and iron, the outer border of the iron region can be determined arbitrarily, as long as magnetic saturation is avoided. We choose a circular external border which encloses the design area. The result of

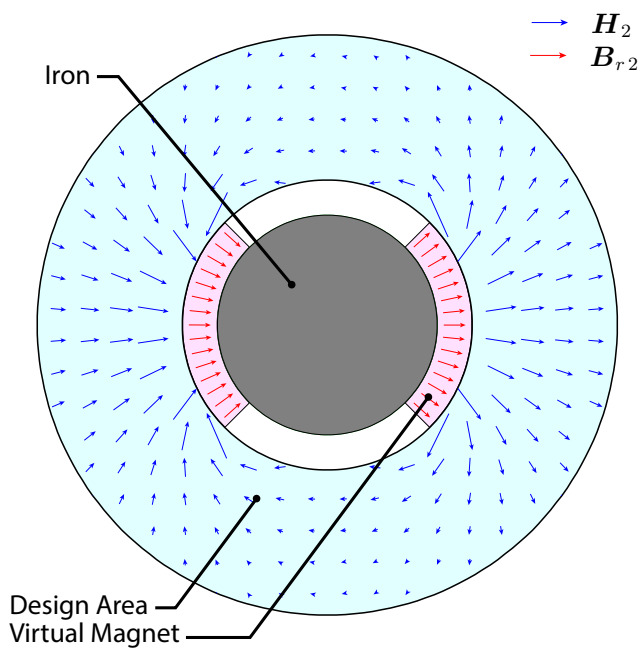

(a)

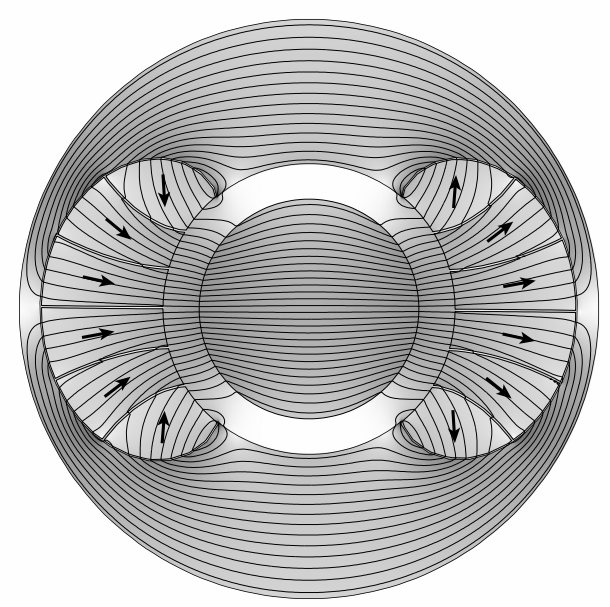

(b)

Figure 4: Design of a magnetic assembly for application in magnetic refrigeration. The purpose of the magnetic system is to create high and low field regions in the air gap between the iron core and the external cylinder. Fig. 4(a): geometry of the virtual system. Fig. 4(b): FEM simulation of the optimal segmentation.

the FEM simulation is shown in Fig. 4(b). The flux density norm averaged over the high field region is equal to $1.25 \mathrm{~T}$ and averaged over the low field region is $0.13 \mathrm{~T}$, which is a satisfactory result with respect to other published magnetic refrigeration devices [23]. The norm of the flux density evaluated at the middle radial position of the air gap is plotted in Fig. 5 as function of the angular coordinate $\phi$ in the interval $\phi \in\left[-90^{\circ}, 90^{\circ}\right]$. It would be possible to further reduce the average norm in the low field region by employing non-linear optimization techniques as the final step of the optimization process. When the field is calculated with the more realistic models for magnets and iron, the flux density norm averaged over the high field region decreases slightly to $1.23 \mathrm{~T}$, while the low field region average remains $0.13 \mathrm{~T}$.

\section{Conclusions}

We have briefly introduced a new method for optimal segmentation of magnetic systems, based on a linear objective functional. Its usefulness has been illustrated for three different applications of permanent magnet arrays.

Since the virtual field can be computed by means of FEM, this technique is applicable to any geometry, even when the analytical solution is not known.

The solution is globally optimal with respect to the considered geometry and objective functional. Moreover, the method can be implemented into a fast algorithm, since only one FEM computation is necessary. 


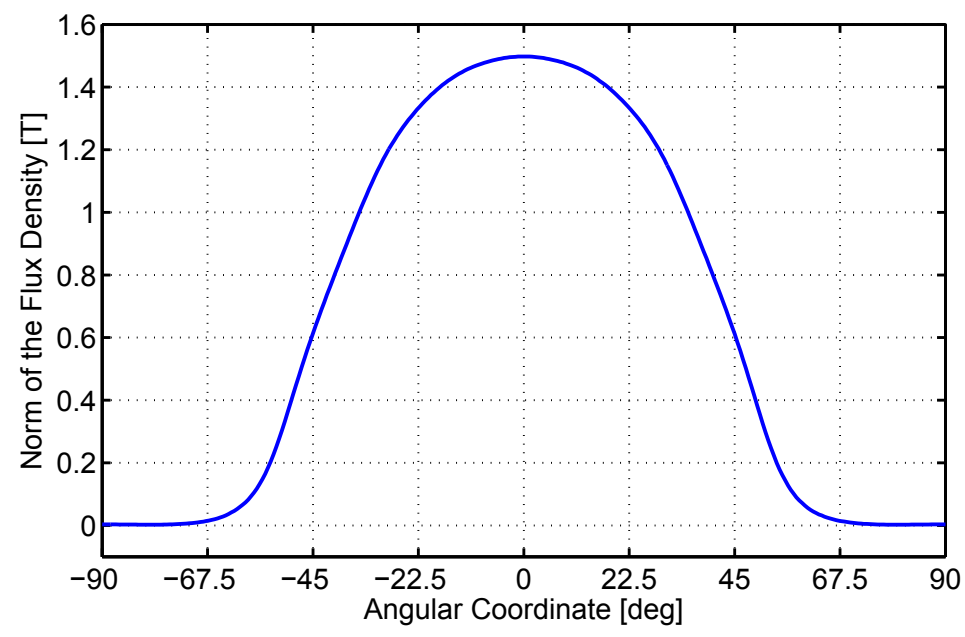

Figure 5: Norm of the air gap flux density for the magnetic refrigeration example. The objective is to maximize the field in the interval $\phi \in\left[-45^{\circ}, 45^{\circ}\right]$

It would be interesting to extend our approach by considering its generalization to the case of a non-linear objective functional. This problem could be solved with an iterative procedure involving the linear approximation to the objective functional at each step.

\section{Acknowledgment}

This work was financed by the ENOVHEAT project which is funded by Innovation Fund Denmark (contract no. 12-132673).

\section{References}

[1] O. Gutfleisch, M. A. Willard, E. Brück, C. H. Chen, S.G. Sankar and J.P. Liu, Magnetic materials and devices for the 21st century: stronger, lighter, and more energy efficient, Adv. Mater. 23 (7), 821-42 (2011).

[2] J.M.D. Coey, Permanent magnet applications, J. Magn. Magn. Mater. 248, 441-456 (2002).

[3] J. S. Choi, J. Yoo, S. Nishiwaki and K. Izui, Optimization of Magnetization Directions in a 3-D Magnetic Structure, IEEE Trans. Magn. 46 (6), 1603-1606 (2010).

[4] S. Lim, S. Jeong and S. Min, Multi-Component Layout Optimization Method for the Design of a Permanent Magnet Actuator, IEEE Trans. Magn. 52 (3), 7205304, 1-4 (2016).

[5] E. Kuci, F. Henrotte, P. Duysinx, P. Dular and C. Geuzaine, Design Sensitivity Analysis for Shape Optimization of Nonlinear Magnetostatic Systems, IEEE Trans. Magn. 52 (3), 9400904, 1-4 (2016).

[6] S. Cheng and D. P. Arnold, Optimization of Permanent Magnet Assemblies Using Genetic Algorithms, IEEE Trans. Magn. 47 (10), 4104-4107 (2011). 
[7] T. Sato, K. Watanabe and H. Igarashi, Multimaterial Topology Optimization of Electric Machines Based on Normalized Gaussian Network, IEEE Trans. Magn. 51 (3), 7202604, 1-4 (2015).

[8] S. Sato, T. Sato and H. Igarashi, Topology Optimization of Synchronous Reluctance Motor Using Normalized Gaussian Network, IEEE Trans. Magn. 51 (3), 8200904, 1-4 (2015).

[9] B.-K. Son, G.-J. Park, J.-W. Kim, Y.-J. Kim and S.-Y. Jung, Interstellar Search Method With Mesh Adaptive Direct Search for Optimal Design of Brushless DC Motor, IEEE Trans. Magn. 52 (3), 8201004, 1-4 (2016).

[10] M. Fasil, N. Mijatovic, B. B. Jensen, and J. Holboll, Finite-Element Model-Based Design Synthesis of Axial Flux PMBLDC Motors, IEEE Trans. Appl. Supercond. 26 (4), 0602905, $1-5(2016)$.

[11] L. Wu and Z.-Q. Zhu, Analytical Modeling of Surface-Mounted PM Machines Accounting for Magnet Shaping and Varied Magnet Property Distribution, IEEE Trans. Magn. 50 (7), 8101511, 1-11 (2014).

[12] T. Shi, Z. Qiao, C. Xia, H. Li and Z. Song, Modeling, Analyzing, and Parameter Design of the Magnetic Field of a Segmented Halbach Cylinder, IEEE Trans. Magn. 48 (5), 1890-1898 (2012).

[13] M. Markovic and Y. Perriard, Optimization Design of a Segmented Halbach PermanentMagnet Motor Using an Analytical Model, IEEE Trans. Magn. 45 (7), 2955-2960 (2009).

[14] D.-K. Lim, K.-P. Yi, D.-K. Woo, H.-K. Yeo, J.-S. Ro, C.-G. Lee and H.-K. Jung, Analysis and Design of a Multi-Layered and Multi-Segmented Interior Permanent Magnet Motor by Using an Analytic Method, IEEE Trans. Magn. 50 (6), 8201308, 1-8 (2014).

[15] W.F. Brown, Magnetostatic principles in ferromagnetism, North-Holland Publishing Company, Amsterdam, (1962).

[16] N.I. Klevets, Synthesis of magnetic systems producing field with maximal scalar characteristics, J. Magn. Magn. Mater. 285, 401-409 (2005).

[17] N.I. Klevets, Optimal design of magnetic systems, J. Magn. Magn. Mater. 306, 281-291 (2006).

[18] J. H. Jensen and M. G. Abele, Maximally efficient permanent magnet structures, J. Appl. Phys. 79 (2), 1157-1163 (1996).

[19] A. R. Insinga, R. Bjørk, A. Smith, and C. R. H. Bahl, Globally Optimal Segmentation of Permanent-Magnet Systems, Phys. Rev. Applied 5 (6), 064014, 1-16 (2016).

[20] Y. Sato, Piecewise linear approximation of planar curves by perimeter optimization, Pattern Recognition 25 (12), 1535-1543 (1992).

[21] A. R. Insinga, C.R.H. Bahl, R. Bjørk and A. Smith, Performance of Halbach magnet arrays with finite coercivity, J. Magn. Magn. Mater. 40, 369-376 (2016).

[22] B. Biswas, A magnetic quadrupole from rectangular permanent magnets, Nucl. Instrum. Methods Phys. Res., Sect. A 605, 233-242 (2009).

[23] R. Bjørk, C. R. H. Bahl, A. Smith and N. Pryds, Improving Magnet Designs With High and Low Field Regions, IEEE Trans. Magn. 47 (6), 1687-1692 (2011). 
[24] R. Bjørk, C. R. H. Bahl, A. Smith and N. Pryds, Review and comparison of magnet designs for magnetic refrigeration, Int. J. Refrigeration 33 (3), 437-448 (2010). 